Pacific Journal of Mathematics

ENTROPY OF AUTOMORPHISMS ON L.C.A. GROUPS 


\title{
ENTROPY OF AUTOMORPHISMS ON L.C.A. GROUPS
}

\author{
Justin Peters
}

In this paper we will consider entropy of automorphisms on locally compact abelian groups. Bowen's definition of entropy of a uniformly continuous mapping applies in particular to topological automorphisms of l.c.a. groups. If $h_{B}(\alpha, G)$ denotes the Bowen entropy of $\alpha \in \operatorname{Aut}(G)$, we investigate the appropriate dual notion $h_{\infty}(\hat{\alpha}, \hat{G})$ of the adjoint automorphism $\hat{\alpha}$ on the dual group $\hat{G}$, and show $h_{B}(\alpha, G)=$ $h_{\infty}(\hat{\alpha}, \hat{G})$. We define the total entropy $h(\alpha, G)$ of $\alpha$ on $G$ to be the sum $h_{B}(\alpha, G)+h_{\infty}(\alpha, G)$ and show that with this definition, $h(\alpha, G)$ coincides with Kolmogorov-Sinai entropy if $G$ is compact and furthermore the invariance properties present in the compact case are retained for an arbitrary l.c.a. group $G$. We also obtain the addition theorem for entropy and a formula for the entropy on projective limits. In conclusion we mention some questions which arise.

o. Let $G$ be a locally compact abelian group (l.c.a. group) and $\operatorname{Aut}(G)$ the group of topological automorphisms of $G$, i.e., those automorphisms which are also homeomorphisms of $G$. If $\Gamma$ is the dual group of $G$ and $\alpha \in \operatorname{Aut}(G)$, the adjoint automorphism $\hat{\alpha}$ is defined by $\hat{\alpha}(\tau(x))=\tau\left(\alpha^{-1}(x)\right), \tau \in \Gamma, x \in G$. $\hat{\alpha}$ is in $\operatorname{Aut}(\Gamma)$, and in fact $\alpha \rightarrow \hat{\alpha}$ describes an antiisomorphism of $\operatorname{Aut}(G)$ to $\operatorname{Aut}(\Gamma)$. Consider the following properties which we would like an entropy function $h(\alpha, G)$ to possess:

(i) if $G$ is compact, $h(\alpha, G)$ is the Kolmogorov-Sinai entropy of $\alpha$ with respect to haar measure; in general $h(\alpha, G) \geqq h_{B}(\alpha, G)$, the Bowen entropy.

(ii) $h\left(\alpha^{k}, G\right)=k \cdot h(\alpha, G), k$ a positive integer;

(iii) if $\alpha_{1}, \alpha_{2} \in \operatorname{Aut}(G)$ are conjugate (i.e., there exists $\beta \in \operatorname{Aut}(G)$ with $\left.\beta \alpha_{1}=\alpha_{2} \beta\right)$, then $h\left(\alpha_{1}, G\right)=h\left(\alpha_{2}, G\right)$;

(iv) if $G_{i}$ is a 1.c.a. group and $\alpha_{i} \in \operatorname{Aut}\left(G_{i}\right), i=1,2$, then $h\left(\alpha_{1} \times \alpha_{2}, G_{1} \times G_{2}\right)=h\left(\alpha_{1}, G_{1}\right)+h\left(\alpha_{2}, G_{2}\right)$;

( v ) $h(\alpha, G)=h\left(\alpha^{-1}, G\right)$;

(vi) $h(\alpha, G)=h(\hat{\alpha}, \Gamma)$;

(vii) $h(\theta, G)=0, \theta$ the identity map.

1. Before continuing, let us observe that in the class of compactly generated abelian Lie groups, there is a unique smallest function satisfying (i) through (vii). For any such Lie group $G$ can be written as $\boldsymbol{R}^{n} \times Z^{m} \times K, K$ a compact Lie group, and so any $\alpha \epsilon$ $\operatorname{Aut}(G)$ has a corresponding decomposition as $\alpha_{1} \times \alpha_{2} \times \alpha_{3}$. By (iv), 
$h(\alpha, G)=h\left(\alpha_{1}, \boldsymbol{R}^{n}\right)+\left(\alpha_{2}, Z^{m}\right)+h\left(\alpha_{3}, K\right)$. Now $h\left(\alpha_{3}, K\right)$ is determined by (i), and $h\left(\alpha_{2}, Z^{m}\right)=h\left(\hat{\alpha}_{2}, T^{m}\right)$ is determined by (vi) and (i). $\alpha_{1}$ is conjugate in $\operatorname{Aut}\left(\boldsymbol{R}^{n}\right)=\mathrm{GL}(n, \boldsymbol{R})$ to a linear transformation $A=$ $A_{1} \times \cdots \times A_{l}$, where each $A_{i}$ has at most two eigenvalues, $\lambda_{i}, \bar{\lambda}_{i}$ (not counting multiplicities). By (iii) and (iv) $h\left(\alpha_{1}, \boldsymbol{R}^{n}\right)=h\left(A, \boldsymbol{R}^{n}\right)=$ $\sum_{i=1}^{l} h\left(A_{i}, R^{k_{i}}\right)$, where $k_{i}$ is the dimension of the subspace corresponding to $A_{i}$. Set $\varepsilon_{i}=\left\{\begin{array}{r}1 \text { if }\left|\lambda_{i}\right| \geqq 1 \\ -1 \text { if }\left|\lambda_{i}\right|<1\end{array}\right.$. By (v), $h\left(A, \boldsymbol{R}^{n}\right)=\sum_{i=1}^{l} h\left(A^{\varepsilon_{i}}, \boldsymbol{R}^{k_{2}}\right)$. Now the Bowen entropy $h_{B}\left(A_{i}^{\varepsilon_{i}}, R^{k_{i}}\right)=k_{i}|\log | \lambda_{i}||$ [1; Theorem 15]. Thus by (i), $h\left(\alpha_{1}, \boldsymbol{R}^{n}\right) \geqq \sum_{i=1}^{l} k_{i}|\log | \lambda_{i}||$. In fact if we set $h\left(\alpha_{1}, \boldsymbol{R}^{n}\right)=$ $\sum_{i=1}^{l} k_{i}|\log | \lambda_{i}||$, it is easy to check that (i)-(vii) are satisfied.

2. We now recall Bowen's definition of topological entropy, recast in the slightly more general context of uniform spaces, which has the advantage that we need not restrict ourselves to metric l.c.a. groups, without complicating the proofs. Let $(X, \mathscr{U})$ be a uniform space and $T: X \rightarrow X$ uniformly continuous. A set $E \subset X$ is $(n, U)$-separated $(U \in \mathscr{Q})$ if for any distinct $x, y \in E$ there is a $j$ such that $0 \leqq j<n$ and $\left(T^{j}(x), T^{j}(y)\right) \notin U$. A set $F$ is said to $(n, U)$ span another set $K$ (with respect to $T$ ) provided that for each $x \in K$ there is a $y \in F$ for which $\left(T^{j}(x), T^{j}(y)\right) \in U$ for all $0 \leqq j<n$.

For a compact set $K \subset X$ let $r_{n}(U, K)$ (where $U \in \mathscr{C}$ ) be the smallest cardinality of any $(n, U)$ spanning set $F$ for $K$ (with repect to $T$ ) and let $s_{n}(U, K)$ denote the largest cardinality of any $(n, U)$-separated set $E$ contained in $K$. Define

$$
\bar{r}_{T}(U, K)=\limsup _{n \rightarrow \infty} \frac{1}{n} \log r_{n}(U, K)
$$

and

$$
\bar{s}_{T}(U, K)=\limsup _{n \rightarrow \infty} \frac{1}{n} \log s_{n}(U, K) .
$$

It can be shown that

$$
r_{n}(U, K) \leqq s_{n}(U, K) \leqq r_{n}(V, K)<\infty
$$

if $V \circ V \subset U$; also if $U_{1} \subset U_{2}$

$$
\bar{r}_{T}\left(U_{1}, K\right) \geqq \bar{r}_{T}\left(U_{2}, K\right) \text { and } \bar{s}_{T}\left(U_{1}, K\right) \geqq \bar{s}_{T}\left(U_{2}, K\right) \text {. }
$$

Finally set $h_{B}(T, K, X)=\lim _{U \in \varkappa} \bar{r}_{T}(U, K)$, and

$$
h_{B}(T, X)=\sup _{K \text { compact }} h_{B}(T, K, X) \text {. }
$$

In our context $X$ will always be a l.c.a. group $G$ and the uniformity $\mathscr{C}$ will be the usual left (right, two-sided) uniformity on $G$.

3. We wish to introduce a second invariant. In doing so, however, we will restrict our attention to automorphisms of a l.c.a. 
group $G$. Let $\alpha \in \operatorname{Aut}(G)$ and $U \subset G$ a precompact neighborhood of the identity. Set

$$
U_{\alpha, n}=U+\alpha^{-1} U+\cdots+\alpha^{-(n-1)} U
$$

and

$$
h_{\infty}(\alpha, U, G)=\lim _{n} \sup \frac{1}{n} \log \mu\left(U_{\alpha, n}\right),
$$

where $\mu$ is a fixed haar measure on $G$. Finally, set

$$
h(\alpha, G)=\lim _{U} k(\alpha, U, G),
$$

where the net $\{U\}$ is directed by $U_{1}<U_{2}$ iff $U_{1} \subset U_{2}$.

Note that the value of $h_{\infty}(\alpha, G)$ is independent of the particular choice of haar measure. $h_{\infty}(\alpha, G)$ was considered in [5] in the context of discrete groups.

4. THEOREM. Let $G$ be a locally compact abelian group and $\alpha \in \operatorname{Aut}(G)$. The function $h(\alpha, G)$ defined by $h(\alpha, G)=h_{B}(\alpha, G)+$ $h_{\infty}(\alpha, G)$ satisfies properties (i) through (vii) above.

5. REMARK. $h_{B}(\alpha, G)$ can also be computed as follows:

$$
h_{B}(\alpha, G)=\lim _{U} \lim \sup _{n}-\frac{1}{n} \log \mu\left(\bigcap_{j=0}^{n-1} \alpha^{-j} U\right),
$$

where $\mu$ is a haar measure on $G$, and the outer limit is taken over a precompact neighborhood base $\{U\}$ converging to the identity [1; Proposition 7].

6. EXAmple. The $p$-adic shift. Let $\Omega_{p}$ be the $p$-adic group; $\Omega_{p}=\left\{\bar{x}=\left(\cdots, x_{k}, x_{k+1}, \cdots\right): x_{k} \in\{0,1, \cdots, p-1\},-\infty<k<\infty\right.$, and $x_{k}=0$ for all $k<n_{0}$, where $n_{0}$ is an integer depending on $\left.\bar{x}\right\}$. The group operation and haar measure on $\Omega_{p}$ are described, for example, in [2]. Let $\alpha \in \operatorname{Aut}\left(\Omega_{p}\right)$ be the bilateral shift given by $\alpha(\bar{x})=\bar{y}$, where $y_{k}=x_{k-1},-\infty<k<\infty$. It is easily seen that for any measurable set $E \subset \Omega_{p}$, the haar measure $m(\alpha E)=p m(E)$, and so $\alpha$ is a nonunimodular automorphism with modular function $\Delta(\alpha)=p$. Let $U_{n}=\left\{\bar{x} \in \Omega_{p}: x_{k}=0\right.$ for $\left.k<n\right\}$. Then $\left\{U_{n}: n=0,1, \cdots\right\}$ forms a neighborhood base at 0 in $\Omega_{p}$. We normalize the haar measure $\mu$ so that $\mu\left(U_{0}\right)=1 . \quad h_{B}\left(\alpha, U_{n}\right)=\lim _{k \rightarrow \infty}-1 / k \log \mu\left(\bigcap_{j=0}^{k-1} \alpha^{-j} U_{n}\right)=$ $\lim _{k \rightarrow \infty}-1 / k \log \mu\left(U_{n+k}\right)=\lim _{k \rightarrow \infty}-1 / k \log p^{-(n+k)}=\log p$. Since this is independent of $n, h_{B}\left(\alpha, \Omega_{p}\right)=\log p$.

To compute $h_{\infty}\left(\alpha, \Omega_{p}\right)$, once again it is sufficient to consider the sets $U_{n}, n \in Z$, since any precompact set $E$ is contained in some $U_{n}$. Since $\alpha^{-1} U_{n}=U_{n+1} \subset U_{n}$, for any $k \in Z^{+}$we have 


$$
\mu\left(U_{n}+\alpha^{-1} U_{n}+\cdots+\alpha^{-(k-1)} U_{n}\right) \leqq \mu\left(k U_{n}\right)
$$

As haar measure on locally compact abelian groups has polynomial growth, we conclude that

$$
\begin{aligned}
h_{\infty}\left(\alpha, \Omega_{p}, U_{n}\right) & =\lim _{k \rightarrow \infty} \frac{1}{k} \log \mu\left(U_{n}+\alpha^{-1} U_{n}+\cdots+\alpha^{-(k-1)} U_{n}\right) \\
& =0, \text { and consequently } \\
h_{\infty}\left(\alpha, \Omega_{p}\right)=0 & .
\end{aligned}
$$

Consider now $\alpha^{-1} \cdot \alpha^{-1} \bar{x}=\bar{y}$, where $y_{k}=x_{k+1}, k \in Z$. Since $\alpha U_{n}=$ $U_{n-1} \supset U_{n}, \bigcap_{j=0}^{k-1} \alpha^{j} U_{n}=U_{n}$. It follows that $h_{B}\left(\alpha^{-1}, \Omega_{p}\right)=0$. On the other hand, observe that

$$
\alpha^{k-1} U_{n} \subset U_{n}+\alpha U_{n}+\cdots+\alpha^{k-1} U_{n} \subset \alpha^{k} U_{n} .
$$

(The right hand containment is easily verified by induction.) Thus $1 / k \log p^{k-1-n} \leqq 1 / k \log \mu\left(U_{n}+\alpha U_{n}+\cdots+\alpha^{k-1} U_{n}\right) \leqq 1 / k \log p^{k-n}$. Hence $h_{\infty}\left(\alpha^{-1}, \Omega_{p}\right)=\log p$.

Next, recall that the dual group $\Omega_{p}^{*}$ of $\Omega_{p}$ is given by $\Omega_{p}^{*}=$ $\left\{\bar{x}=\left(\cdots, x_{k}, x_{k+1}, \cdots\right): x_{k} \in\{0,1, \cdots, p-1\}\right.$, and $x_{k}=0$ for all $k>n$, where $n$ depends only on $\bar{x}\}$. The group operation and haar measure on $\Omega_{p}^{*}$ are analogous to those of $\Omega_{p}$. The adjoint $\hat{\alpha}$ is given by $\hat{\alpha}(\bar{x})=\bar{y}$, where $y_{k}=x_{k-1}$. It is easy to compute that $h_{B}\left(\hat{\alpha}, \Omega_{p}^{*}\right)=0$, $h_{\infty}\left(\hat{\alpha}, \Omega_{p}^{*}\right)=\log p, h_{B}\left(\hat{\alpha}^{-1}, \Omega_{p}^{*}\right)=\log p$, and $h_{\infty}\left(\hat{\alpha}^{-1}, \Omega_{p}^{*}\right)=0$. We see that $h\left(\alpha, \Omega_{p}\right)=h\left(\alpha^{-1}, \Omega_{p}\right)=h\left(\hat{\alpha}, \Omega_{p}^{*}\right)=h\left(\hat{\alpha}^{-1}, \Omega_{p}^{*}\right)=\log p$.

The situation above contrasts with that of the bilateral shift $\beta$ on $G=\prod_{j=-\infty}^{\infty}\left(Z_{p}\right)_{i},\left(\left(Z_{p}\right)_{i}=Z_{p}\right.$ for all $\left.i \in Z\right)$, with dual group $\Gamma=$ $\sum_{j=-\infty}^{\infty}\left(Z_{p}\right)_{j}$. Here $h_{B}(\beta, G)=h_{B}\left(\beta^{-1}, G\right)=\log p$ by the KolmogorovSinai theorem. $h_{\infty}(\beta, G)=h_{\infty}\left(\beta^{-1}, G\right)=0 . \quad$ Also $h_{B}(\widehat{\beta}, \Gamma)=h_{B}\left(\widehat{\beta}^{-1}, \Gamma\right)=$ 0 and $h_{\infty}(\widehat{\beta}, \Gamma)=h_{\infty}\left(\widehat{\beta}^{-1}, \Gamma\right)=\log p$.

7. EXAMPLE. We verify directly that if $\alpha \in \operatorname{Aut}\left(\boldsymbol{R}^{n}\right)=\mathrm{GL}(n, \boldsymbol{R})$, then $h\left(\alpha, \boldsymbol{R}^{n}\right)=\sum_{i=1}^{l} k_{i}|\log | \lambda_{i}||$, where $\left\{\lambda_{1}, \cdots, \lambda_{l}\right\}$ are eigenvalues of $\alpha$ and $k_{i}$ is the multiplicity of $\lambda_{i}$. (Here our notation differs from that of $\S 1$.) Now $\alpha$ gives rise to $\tilde{\alpha} \in \mathrm{GL}(n, C)$ by $\tilde{\alpha}(x+i y)=$ $\alpha(x)+i \alpha(y), x, y \in \boldsymbol{R}^{n}$. Since $\tilde{\alpha}$ can be identified with $\alpha \times \alpha$ on $\boldsymbol{R}^{2 n}$, the effect of changing the base field from $\boldsymbol{R}$ to $\boldsymbol{C}$ is to double the entropy. If we can show $h\left(\widetilde{\alpha}, C^{n}\right)=\sum_{i=1}^{l} 2 k_{i}|\log | \lambda_{i}||$, the formula above will follow. There is a basis for $C^{n}$ such that the matrix $A$ of $\tilde{\alpha}$ is in Jordan canonical form: thus $A=A_{1} \times \cdots \times A_{l}$ and each $A_{i}$ is a $k_{i} \times k_{i}$ Jordan block with eigenvalue $\lambda_{i}$.

Let $B$ be a $k \times k$ matrix of the form $B=\left[\begin{array}{rrrrr}\lambda & 1 & 0 & \cdots & 0 \\ 0 & \lambda & 1 & \cdots & 0 \\ \vdots & & & \vdots \\ 0 & & & & \\ 0 & & \end{array}\right]$ with 
$\lambda \neq 0$ on the diagonal and 1 on the superdiagonal. Then $B^{m}$ is an upper triangular matrix with $\lambda^{m}$ on the diagonal and $1 / j !\left(d^{(j)} / d \lambda^{j}\right)\left(\lambda^{m}\right)$ on the $j$ th superdiagonal, $j=1,2, \cdots, k-1, m=1,2, \cdots$. A crude estimate shows

$$
\left\|B^{m} x\right\| \leqq\left\{\begin{array}{lll}
k m^{k}\|x\|, & \text { if } & |\lambda| \leqq 1 \\
|\lambda|^{m} k m^{k}\|x\|, & \text { if } & |\lambda|>1
\end{array}\right.
$$

Thus, if $U$ is a ball in $C^{k}$ and $|\lambda| \leqq 1$,

$$
\begin{gathered}
U+B U+\cdots+B^{N-1} U \\
\subset U+k U+\cdots+k(N-1)^{k} U
\end{gathered}
$$

$\subset k^{2} N^{k} U$ for every positive integer $N$.

Since haar measure $\mu$ on $C^{k}$ has polynomial growth, $1 / N \log \left(k^{2} N^{k} U\right) \rightarrow 0$ as $N \rightarrow \infty$. So if $|\lambda| \leqq 1, h_{\infty}\left(B^{1}, C^{k}\right)=0$.

If $|\lambda|>1$,

$$
\begin{gathered}
U+B U+\cdots+B^{N-1} U \\
\subset U+|\lambda| k U+\cdots+|\lambda|^{N-1} k(N-1)^{k} U \\
\subset\left(1+|\lambda|+\cdots+|\lambda|^{N-1}\right) k N^{k} U \\
\subset \frac{|\lambda|^{N}-1}{|\lambda|-1} k N^{k} U .
\end{gathered}
$$

The polynomial growth condition on $\mu$ means that for any convex neighborhood $V$ of $O$ in $C^{k}$ there is a $c>0$ and an exponent $q$ such that $\mu(t V) \leqq c t^{q}, t>0$. In fact we can take $q=2 k$. Thus

$$
\mu\left(B^{N-1} U\right) \leqq \mu\left(U+B U+\cdots+B^{N-1} U\right) \leqq \mu\left(\frac{|\lambda|^{N}-1}{|\lambda|-1} k N^{k} U\right) .
$$

Since $\mu\left(B^{N-1} U\right)=\Delta(B)^{N-1} \mu(U)$, where $\Delta(B)=|\lambda|^{2 k}$ is the modular function, we have

$$
|\lambda|^{2 k(N-1)} \leqq \mu\left(U+B U+\cdots+B^{N-1} U\right) \leqq c\left(\frac{|\lambda|^{N}-1}{|\lambda|-1}\right)^{2 k} k^{2 k} N^{2 k^{2}} .
$$

Taking the log of both sides and dividing by $N$ and taking the limit as $N \rightarrow \infty$ we get that $h_{\infty}\left(B^{-1}, C^{k}\right)=2 k \log |\lambda|$.

From (8.b) we have

$$
h_{\infty}\left(B, C^{k}\right)=h_{\infty}\left(B^{-1}, C^{k}\right)-2 k \log |\lambda| .
$$

We conclude $h_{\infty}\left(B, C^{k}\right)=\left\{\begin{array}{l}0, \\ -2 k \log |\lambda|,|\lambda| \geqq 1 . \\ |\lambda|<1 .\end{array}\right.$ From [1; Theorem 15] $h_{\infty}\left(\tilde{\alpha}, C^{n}\right)=\left.\sum\right|_{\lambda_{i} \mid>1} 2 k_{i} \log \left|\lambda_{i}\right| . \quad h\left(\widetilde{\alpha}, C^{n}\right)=\sum_{i=1}^{i} 2 k_{i}|\log | \lambda_{i}||$ follows.

8. We now turn to the proof of Theorem 4. Property (i) fol- 
lows from the definition of $h(\alpha, G)$, while (ii), (iii), (iv) and (vill) need only be verified for $h_{\infty}$, since they are known to hold for $h_{B}([1])$. The verifications are straightforward and are omitted. Actually, in $\S 9$ we will show $h_{\infty}(\alpha, G)=h_{B}(\hat{\alpha}, \widehat{G})$, and so these properties of $h_{\infty}$ will in fact follow from those of $h_{B}$. Note that (vii) follows since l.c.a. groups have polynomial growth.

Recall that the modular function $\Delta(\alpha)(\alpha \in \operatorname{Aut}(G))$ is defined by

$$
\mu(\alpha U)=\Delta(\alpha) \mu(U),
$$

where $U$ is any measurable subset of $G$. Equivalently, for $f \in$ $L^{1}(G)$,

$$
\int_{G} f \circ \alpha d \mu=\Delta(\alpha)^{-1} \int_{G} f d \mu .
$$

To show (v) we will show that

(a) $h_{B}(\alpha, G)=h_{B}\left(\alpha^{-1}, G\right)+\log \Delta(\alpha)$ and

(b) $h_{\infty}(\alpha, G)=h_{\infty}\left(\alpha^{-1}, G\right)-\log \Delta(\alpha)$.

If $U$ is a precompact neighborhood of the identity in $G$ and $\mu$ is haar measure we have $\mu\left(\bigcap_{j=0}^{n-1} \alpha^{-j} U\right)=\mu\left(\alpha^{-(n-1)} \bigcap_{j=0}^{n-1} \alpha^{j} U\right)=$ $\Delta(\alpha)^{-(n-1)} \mu\left(\bigcap_{j=0}^{n-1} \alpha^{j} U\right)(\alpha \in \operatorname{Aut}(G))$, and (a) follows. For (b) notice $\mu\left(U+\alpha^{-1} U+\cdots+\alpha^{-(n-1)} U\right)=\mu\left(\alpha^{-(n-1)}\left(U+\alpha U+\cdots+\alpha^{(n-1)} U\right)\right)=$ $\Delta(\alpha)^{-(n-1)} \mu\left(U+\mu U+\cdots+\alpha^{(n-1)} U\right)$.

9. To show $h(\alpha, G)=h(\hat{\alpha}, \hat{G})$ we will prove $h_{\infty}(\alpha, G)=h_{B}(\hat{\alpha}, \hat{G})$, as mentioned above. This, together with the Pontryagain-van Kampen duality theorem, will yield the assertion.

The strategy of the proof is to rewrite the defining expressions for $h_{B}$ and $h_{\infty}$ as limits of convolutions of characteristic functions of sets, and then replace characteristic functions with nonnegative positive definite $L^{1}$-functions, which is self-dual under the Fourier transform. It will be convenient to introduce some notations. If $\phi$ is a function on $G$ and $\alpha \in \operatorname{Aut}(G), \alpha \phi$ will denote the function $(\alpha \phi)(x)=\phi\left(\alpha^{-1} x\right), x \in G$. Also, we will let

$$
C_{n}(\phi, \alpha)=\phi *\left(\alpha^{-1} \phi\right) * \cdots *\left(\alpha^{-(n-1)} \phi\right), n>0 .
$$

LEMMA 9.1. Let $n \geqq 1$ and $U_{i}=-U_{i}$ be symmetric neighborhoods of $0,1 \leqq i \leqq n$. Then

$$
\frac{\mu\left(U_{1}\right)^{2} \mu\left(U_{2}\right)^{2} \cdots \mu\left(U_{n}\right)^{2}}{\chi_{U_{1}} * \chi_{U_{1}} * \chi_{U_{2}} * \chi_{U_{2}} * \cdots * \chi_{U_{n}} * \chi_{U_{n}}(0)} \leqq \mu\left(2 U_{1}+2 U_{2}+\cdots+2 U_{n}\right) .
$$

Proof. The convolution of characteristic functions $\chi_{U_{i}} * \chi_{U_{i}}$ is positive definite, $1 \leqq i \leqq n$, since $\chi_{U_{i}}^{*}(x)=\chi_{U_{i}}(-x)^{-}=\chi_{U_{i}}(x)$. Thus 
$\chi_{U_{1}} * \chi_{U_{1}} * \chi_{U_{2}} * \chi_{U_{2}} * \cdots * \chi_{U_{n}} * \chi_{U_{n}}$ is positive definite, and achieves its maximum at 0 . Now

$$
\begin{aligned}
& \mu\left(U_{i}\right)^{2} \mu\left(U_{2}\right)^{2} \cdots \mu\left(U_{n}\right)^{2}=\int_{G} \chi_{U_{1}} * \chi_{U_{1}} * \chi_{U_{2}} * \chi_{U_{2}} * \cdots * \chi_{U_{n}} * \chi_{U_{n}}(x) d \mu(x) \\
& \leqq \chi_{U_{1}} * \chi_{U_{1}} * \chi_{U_{2}} * \chi_{U_{2}} * \cdots * \chi_{U_{n}} * \chi_{U_{n}}(0) \mu\left(2 U_{1}+2 U_{2}+\cdots+2 U_{n}\right),
\end{aligned}
$$

since the integrand is supported in $2 U_{1}+2 U_{2}+\cdots+2 U_{1}$.

Lemma 9.2. Let $U_{i}, 1 \leqq i \leqq n$ be as in Lemma 9.1 and $r$ a positive integer. Then

$$
\mu\left(2 U_{1}+2 U_{2}+\cdots+2 U_{n}\right) \leqq \frac{\mu\left((r+1) U_{2}\right)^{2}\left((r+1) U_{2}\right)^{2} \cdots\left((r+1) U_{n}\right)^{2}}{\chi_{r U_{1}} * \chi_{r U_{1}} * \cdots * \chi_{r U_{n}} * \chi_{r U_{n}}(0)} .
$$

Proof. Let $\lambda_{x}$ be the point mass at $x$, so that $\lambda_{x} * f(z)=f(z-x)$. Let $u_{i}, u_{i}^{\prime} \in U_{i}, 1 \leqq i \leqq n$, and $x=u_{1}+u_{1}^{\prime}+\cdots+u_{n}+u_{n}^{\prime} \in 2 U_{1}+\cdots+$ $2 U_{n}$. We have

$$
\begin{aligned}
\chi_{(r+1) U_{1}} & \chi_{(r+1) U_{1}} * \cdots * \chi_{(r+1) U_{n}} * \chi_{(r+1) U_{n}}(x) \\
& =\lambda_{-x} * \chi_{(r+1) U_{1}} * \chi_{(r+1) U_{1}} * \cdots * \chi_{(r+1) U_{n}} * \chi_{(r+1) U_{n}}(0) \\
& =\left(\lambda_{-u_{1}} * \chi_{(r+1) U_{1}}\right) *\left(\lambda_{-u_{1}^{\prime}} * \chi_{(r+1) U_{1}}\right) * \cdots *\left(\lambda_{-u_{n}} * \chi_{(r+1) U_{n}}\right) *\left(\lambda_{-u_{n}^{\prime}} * \chi_{(r+1) U_{n}}\right)(0) \\
& \geqq \chi_{r U_{1}} * \chi_{r U_{1}} * \cdots * \chi_{r U_{n}} * \chi_{r U_{n}}(0) .
\end{aligned}
$$

Integrating over $2 U_{1}+2 U_{2}+\cdots+2 U_{n}$ gives the result.

LeMmA 9.3. Any precompact neighborhood $V$ of 0 in $G$ is contained in a precompact neighborhood $U$ satisfying

$$
\lim _{r \rightarrow \infty} \frac{\mu((r+1) U)}{\mu(r U)}=1
$$

Proof. Let $H$ be the compactly generated open subgroup generated by $V$. Then $H$ is a projective limit of abelian lie groups [2; Theorem 9.6]; hence it is enough to observe that the assertion holds for $G=R^{n}$ or $G=Z^{m}$, the proofs of which are straightforward and omitted.

Using the notation introduced at the beginning of this section we define

$$
h_{\infty}^{1}(\alpha, G)=\sup _{\substack{U \in G \text { open } \\ \text { precompact }}} \lim _{n} \sup -\frac{1}{n} \log \left\{\frac{\Delta(\alpha)^{n(n-1)}}{\mu(U)^{2 n}} C_{n}\left(\chi_{U} * \chi_{U}, \alpha\right)(0)\right\} .
$$

LEMMA 9.4. $h_{\infty}(\alpha, G)=h_{\infty}^{1}(\alpha, G)$.

Proof. Let $U$ be a precompact open neighborhood of $O$ and 
apply Lemma 9.1 with $U_{j}=\alpha^{-(j-1)} U$. Take the logarithm of both sides of the inequality and divide by $n$; since $\chi_{\alpha-j U}=\alpha^{-j} \chi_{U}$, this yields

$$
\begin{aligned}
- & \frac{1}{n} \log \frac{C_{n}\left(\chi_{U} * \chi_{U}, \alpha\right)(0)}{\mu(U)^{2} \mu\left(\alpha^{-1} U\right)^{2} \cdots \mu\left(\alpha^{-(n-1)} U\right)^{2}} \\
& \leqq \frac{1}{n} \log \mu\left(2 U+\alpha^{-1}(2 U)+\cdots+\alpha^{-(n-1)}(2 U)\right) .
\end{aligned}
$$

From $\mu\left(\alpha^{-j} U\right)=\Delta(\alpha)^{-j} \mu(U)$, we conclude that $h_{\infty}^{1}(\alpha, G) \leqq h_{\infty}(\alpha, G)$.

By Lemma 9.3 we may suppose $U$ satisfies

$$
\lim _{r \rightarrow \infty} \frac{\mu((r+1) U)}{\mu(r U)}=1 .
$$

From Lemma 9.2 we obtain

$$
\begin{aligned}
& \frac{1}{n} \log \mu\left(2 U+\alpha^{-1}(2 U)+\cdots+\alpha^{-(n-1)}(2 U)\right) \\
& \quad \leqq-\frac{1}{n} \log \left\{\frac{\Delta(\alpha)^{n(n-1)}}{\mu(r U)^{2 n}} C_{n}\left(\chi_{r U^{*}} \chi_{r U}, \alpha\right)\right\}+2 \log \frac{\mu((r+1) U)}{\mu(r U)} .
\end{aligned}
$$

It follows from Lemma 9.2 that $h_{\infty}(\alpha, G) \leqq h_{\infty}^{1}(\alpha, G)$ and thus $h_{\infty}(\alpha, G)=h_{\infty}^{1}(\alpha, G)$.

Let $P(G)$ denote the continuous positive definite functions on $G$ with compact support and $C_{00}(G)^{+}$the nonnegative continuous functions on $G$ with compact support. Set

$$
h_{\infty}^{2}(\alpha, G)=\sup _{\phi \in P(G) \cap C_{00}(G)^{\dagger}} \limsup _{n}-\frac{1}{n} \log \left\{\frac{\Delta(\alpha)^{n(n-1) / 2}}{\left(\int_{G} \phi d \mu\right)^{n}} C_{n}(\phi, \alpha)(0)\right\} .
$$

Clearly, $h_{\infty}^{2}(\alpha, G) \geqq h_{\infty}^{1}(\alpha, G)$. On the other hand, by an estimate very similar to the first part of Lemma 9.2 , it follows that $h_{\infty}^{2}(\alpha, G) \leqq$ $h_{\infty}(\alpha, G)$. Thus $h_{\infty}^{2}(\alpha, G)=h_{\infty}^{1}(\alpha, G)$.

Let $L^{1}(G)^{+}$denote the positive cone in $L^{1}(G)=L^{1}(G, \mu)$. Define

$$
h_{\infty}^{3}(\alpha, G)=\sup _{\phi \in P(G) \cap L^{1}(G)^{\dagger}} \lim \sup _{n}-\frac{1}{n} \log \left\{\frac{\Delta(\alpha)^{n(n-1) / 2}}{\left(\int_{G} \phi d \mu\right)^{n}} C_{n}(\dot{\phi}, \alpha)(0)\right\} .
$$

Clearly $h_{\infty}^{3}(\alpha, G) \geqq h_{\infty}^{2}(\alpha, G)$.

Lemma 9.5. $h_{\infty}^{2}(\alpha, G)=h_{\infty}^{3}(\alpha, G)$.

Proof. We sketch the proof briefly, since the details are essentially the same as in [5; Lemma 11] except for the presence of the modular function. First, a routine estimate shows that in the 
definition of $h_{\infty}^{3}$ each $\phi$ which appears inside the brackets \{\} may be replaced by $\phi * \phi$. Then given $\phi \in P(G) \cap L^{1}(G)^{+},\|\dot{\phi}\|_{1}=1$, there is a compact symmetric neighborhood $U$ of $O$ such that if $f=\phi \chi_{U}$, $\|\phi-f\|_{1}<\varepsilon / 2$. If $\psi=f * f, \psi \in P(G) \cap C_{00}(G)^{+}$and

$$
\begin{aligned}
\mid-\frac{1}{n} \log & \left\{\frac{\Delta(\alpha)^{n(n-1) / 2}}{\left(\int_{G} \psi d \mu\right)^{n}} C_{n}(\psi, \alpha)\right\} \\
& +\frac{1}{n} \log \left\{\Delta(\alpha)^{n(n-1) / 2} C_{n}(\phi * \phi, \alpha)\right\} \mid \leqq \log (1-\varepsilon), n=1,2, \cdots .
\end{aligned}
$$

Hence $h_{\infty}^{3}(\alpha, G)=h_{\infty}^{2}(\alpha, G)$.

Suppose now $\Gamma$ is another locally compact abelian group and $\beta \in \operatorname{Aut}(\Gamma)$. As observed in Remark 5, we have

$$
h_{B}(\beta, \Gamma)=\sup _{U} \lim _{n} \sup \left\{-\frac{1}{n} \log \omega\left(\bigcap_{j=1}^{n} \beta^{-j} U\right)\right\}
$$

where $\omega$ is a haar measure on $\Gamma$ and $U$ is a precompact open neighborhood of the neutral element 1 in $\Gamma$. Define

$$
h_{B}^{1}(\beta, \Gamma)=\sup _{\phi \in P(\Gamma) \cap L^{1}(\Gamma)^{+}}-\frac{1}{n}\left\{\log \frac{1}{\phi(e)^{n}} \int_{\Gamma} \phi\left(\beta^{-1} \phi\right) \cdots\left(\beta^{-(n-1)} \phi\right)(y) d \omega(y)\right\} .
$$

LeMma 9.6. $h_{B}^{1}(\beta, \Gamma)=h_{B}(\beta, \Gamma)$.

Proof. Formally the proof is identical to [5; Lemma 12]; the compactness of $\Gamma$, which is assumed there, is not needed.

9.7. Let $G$ be a locally compact abelian group with fixed haar measure $\mu$, and let $\Gamma$ be the dual group with haar measure $\omega$, which is appropriately normalized so that the inversion theorem holds.

We claim that $\widehat{\alpha \phi}=\Delta(\alpha) \hat{\alpha} \hat{\phi}, \phi \in L^{1}(G)$ :

$$
\begin{aligned}
\widehat{\alpha \phi}(\tau) & =\int_{G} \alpha \phi(x) \tau(x)-d \mu(x) \\
& =\int_{G} \phi\left(\alpha^{-1} x\right) \tau(x)-d \mu(x) \\
& =\Delta(\alpha) \int_{G} \phi(x) \tau(\alpha x)-d \mu(x) \\
& =\Delta(\alpha) \int_{G} \phi(x)\left(\hat{\alpha}^{-1} \tau\right)(x)-d \mu(x) \\
& =\Delta(\alpha) \hat{\alpha} \hat{\phi}(\tau) .
\end{aligned}
$$

For $\phi \in P(G) \cap L^{1}(G)^{+}$, 


$$
\begin{aligned}
\frac{\Delta(\alpha)^{n(n-1) / 2}}{\left(\int_{G} \phi d \mu\right)^{n}} C_{n}(\phi, \alpha)(0) & =\frac{\Delta(\alpha)^{n(n-1) / 2}}{(\hat{\phi}(e))^{n}} \int_{\Gamma} \widehat{C_{n}(\hat{\phi}, \alpha)}(y) d \omega(y) \\
& \left.=\frac{\Delta(\alpha)^{n(n-1) / 2}}{(\hat{\phi}(e))^{n}} \int_{\Gamma} \widehat{\phi} \widehat{\left(\alpha^{-1} \phi\right.}\right) \cdots\left(\widehat{\alpha^{-(n-1)}} \phi\right)(y) d \omega(y) \\
& =\frac{1}{(\phi(e))^{n}} \int_{\Gamma} \hat{\phi}\left(\hat{\alpha}^{-1} \hat{\phi}\right) \cdots\left(\hat{\alpha}^{-(n-1)} \hat{\phi}\right)(y) d \omega(y)
\end{aligned}
$$

But by the Fourier Invension Theorem,

$$
\left\{\hat{\phi}: \phi \in P(G) \cap L^{1}(G)^{+}\right\}=P(\Gamma) \cap L^{1}(\Gamma)^{+} .
$$

The above calculation shows that $h_{\infty}^{3}(\alpha, G)=h_{B}^{1}(\hat{\alpha}, \Gamma)$. But $h_{\infty}^{3}(\alpha, G)=$ $h_{\infty}(\alpha, G)$ and $h_{B}^{1}(\hat{\alpha}, \Gamma)=h_{B}(\hat{\alpha}, \Gamma)$. Thus $h_{\infty}(\alpha, G)=h_{B}(\hat{\alpha}, \Gamma)$, as desired.

10. Addition Theorem. Let $G$ be a l.c.a. group, $\alpha \in \operatorname{Aut}(G)$ and $H \subset G$ a closed subgroup such that $\alpha(H)=H$. If $\alpha_{1}$ denotes the restriction of $\alpha$ to $H$ and $\alpha_{2}$ the induced automorphism on the quotient $G / H$, then

$$
h(\alpha, G)=h\left(\alpha_{1}, H\right)+h\left(\alpha_{2}, G / H\right) .
$$

Note. Juzvinskii proves the addition theorem using structure theory in case $G, H$ are compact but not necessarily abelian. The compact case also follows from [1; Theorem 19].

Proof. Let $\phi: G \rightarrow G / H$ be the canonical map and write $\dot{x}$ for $\phi(x)$. Let $K \subset G, C \subset H$ be compact, and $U$ a neighborhood of $O$ in $G$. Suppose $\dot{E}_{2}$ is an $(n, U+H)$-separated set in $\dot{K}$ with respect to $\alpha_{2}$ and $E_{1}$ is an $(n, U \cap H)$-separted set in $C$ with respect to $\alpha_{1}$. For each $\dot{x} \in \dot{E}_{2} \subset \dot{K}$ choose a representative $x \in K$ and denote the set of representatives by $E_{2}$. The set $E=E_{1}+E_{2}=\left\{h+x: h \in E_{1}, x \in\right.$ $\left.E_{2}\right\}$ is $(n, U)$ separated in $K+C$. For suppose $h+x, h^{\prime}+x^{\prime} \in E$, $h+x \neq h^{\prime}+x^{\prime}$. If $x=x^{\prime}$, since $h, h^{\prime}$ are $(n, U \cap H)$-separated there is a $j, 0 \leqq j<n$, with $\alpha_{1}^{-j} h-\alpha_{1}^{-j} h^{\prime} \notin U \cap H$. But then $\alpha^{-j}(x+h)-$ $\alpha^{-j}\left(x^{\prime}+h^{\prime}\right) \notin U$. If $x \neq x^{\prime}$, then $\dot{x} \neq \dot{x}^{\prime}$ and there is a $j, 0 \leqq j<n$, with $\alpha_{2}^{-j} \dot{x}-\alpha_{2}^{-j} \dot{x}^{\prime} \notin U+H$. Hence $\alpha^{-j}(x+h)-\alpha^{-j}\left(x^{\prime}+h^{\prime}\right) \notin U$. In either case, $h+x$ and $h^{\prime}+x^{\prime}$ are $(n, U)$-separated. Thus

$$
s_{n}(U, K+C) \geqq s_{n}(U \cap H, C) s_{n}(U+H, \dot{K}),
$$

and $h_{B}(\alpha, G) \geqq h_{B}\left(\alpha_{1}, H\right)+h_{B}\left(\alpha_{2}, G / H\right)$.

To get the reverse inequality, let $K \subset G$ be compact and $U$ a compact neighborhood of $O$ in $G$. Let $C=(K-K-2 U) \cap H$ and $\dot{F}_{2}$ and $(n, \dot{U})$-spanning set for $\dot{K}$ with respect to $\alpha_{2}$, which we as- 
sume is minimal. Let $F_{1}(n, U \cap H)$-span $C$. For any $y \in K$ there is an $x \in \phi^{-1}\left(\dot{F}_{2}\right)$ with $\alpha^{-j} y-\alpha^{-j} x \in U+H, 0 \leqq j<n$. Equivalently, $y-x \in \bigcap_{j=0}^{n-1} \alpha^{j} U+H$. The minimality assumption on $\dot{F}_{2}$ implies $\dot{F}_{2} \subset \dot{K}+\bigcap_{j=0}^{n-1} \alpha^{j} \dot{U}$. So for each $\dot{x} \in \dot{F}_{2}$ we can choose a representative $x \in K+\bigcap_{j=0}^{n-1} \alpha^{j} U$ and denote the resulting set $F_{2}$. We claim that $F=F_{1}+F_{2}(n, 2 U)$-spans $K$ with respect to $\alpha$. Given $y \in K$ there is an $x \in F_{2}$ and a $u \in \bigcap_{j=0}^{n-1} \alpha^{j} U$ so that $y-x-u \in H$. But $y-x-u \in(K-(K+U)-U) \cap H=(K-K-2 U) \cap H=C$.

Thus there exists $h \in F_{1}$ so that

$$
\alpha^{-j}(y-x-u)-\alpha^{-j} h \in U \cap H, 0 \leqq j<n ;
$$

i.e.,

$$
y-x-u-h \in\left(\bigcap_{j=0}^{n-1} \alpha^{j} U\right) \cap H .
$$

Hence

$$
\begin{gathered}
y-(x+h) \in u+\left(\bigcap_{j=0}^{n-1} \alpha^{j} U\right) \cap H, \\
\subset \bigcap_{j=0}^{n-1} \alpha^{j}(2 U),
\end{gathered}
$$

which shows $F=F_{1}+F_{2}(n, 2 U)$-spans $K$, as claimed. It follows

$$
r_{n}(2 U, K) \leqq r_{n}(\dot{U}, \dot{K}) r_{n}(U \cap H, C),
$$

and consequently $h_{B}(\alpha, G) \leqq h_{B}\left(\alpha_{1}, H\right)+h_{B}\left(\alpha_{2}, G / H\right)$.

We prove the corresponding equality for $h_{\infty}$ by passing to the dual and utilizing the result above. If $K$ is the annihilator of $H$ in $\widehat{G}$, then $K=\widehat{G / H}$ and $\hat{G} / K=\hat{H}$. By the proof of part (vi) of Theorem 4,

$$
\begin{aligned}
h_{\infty}(\alpha, G) & =h_{B}(\widehat{\alpha}, \hat{G}) \\
& =h_{B}\left(\widehat{\alpha}_{1}, \hat{G} / K\right)+h_{B}\left(\hat{\alpha}_{2}, K\right) \\
& =h_{B}\left(\hat{\alpha}_{1}, H\right)+h_{B}\left(\hat{\alpha}_{2}, G / H\right) \\
& =h_{\infty}\left(\alpha_{1}, H\right)+h_{\infty}\left(\alpha_{2}, G / H\right) .
\end{aligned}
$$

CoRollary 11. Suppose $G$ is a projective limit of Lie groups, $G=\operatorname{proj} \lim G_{\nu}, G_{\nu}=G / H_{\nu}, H_{\nu}$ compact and $\alpha$-invariant, and $\alpha_{\nu}$ is the induced automorphism on $G_{\nu}$. Then

$$
h(\alpha, G)=\lim _{\nu} h\left(\alpha_{\nu}, G_{\nu}\right) .
$$

Proof. Set $\alpha^{\nu}$ equal the restriction of $\alpha$ to $H_{\nu}$. First suppose $G$ is compact.

$$
h_{B}(\alpha, G)=h_{\infty}(\hat{\alpha}, \hat{G})=h_{\infty}\left(\hat{\alpha}_{\nu}, \hat{G}_{\nu}\right)+h_{\infty}\left(\hat{\alpha}_{\nu}, \hat{H}_{\nu}\right) .
$$


Now $\hat{G}_{\nu}=K_{\nu}$, the annihilator in $\hat{G}$ of $H_{\nu}$. Furthermore, by properties of projective limits, for any $\tau \in \widehat{G}$ there is a $\nu$ with $H_{\nu} \subset$ kernel $\tau$. Thus $\tau \in K_{\nu}$, and we can write $\widehat{G}=\bigcup_{\nu} K_{\nu}$. Hence, given any finite set $E \subset \hat{G}$ there is a $\nu_{0}$ so that $E \subset K_{\nu}$ for $\nu \geqq \nu_{0}$. By definition, $h(\hat{\alpha}, \hat{G})$ can be approximated arbitrarily closed by taking a sufficiently large finite set $E \subset \widehat{G}$ and forming the limit, $\lim _{n}(1 / n) \log$ $\operatorname{card}\left(E_{\hat{\alpha}, n}\right)$, where $E_{\hat{\alpha}, n}=E+\alpha^{-1} E+\cdots+\hat{\alpha}^{-(n-1)} E . \quad$ Thus $h_{\infty}(\widehat{\alpha}, \widehat{G})=$ $\lim _{\nu} h_{\infty}\left(\hat{\alpha}_{\nu}, K_{\nu}\right)$ i.e., $h_{B}(\alpha, G)=\lim _{\nu} h_{B}\left(\alpha_{\nu}, G_{\nu}\right)$.

Now drop the compactness assumption on $G$ and write

$$
\begin{aligned}
h_{B}(\alpha, G) & =h_{B}\left(\alpha_{\nu}, G_{\nu}\right)+h_{B}\left(\alpha^{\nu}, H_{\nu}\right) \\
& =h_{B}\left(\alpha_{\nu}, G_{\nu}\right)+h_{B}\left(\alpha^{\nu^{\prime}}, H_{\nu^{\prime}}\right)+h_{B}\left(\alpha_{\nu \nu^{\prime}}, H_{\nu} / H_{\nu^{\prime}}\right),
\end{aligned}
$$

for $\nu^{\prime} \geqq \nu$ and $\alpha_{\nu \nu^{\prime}}$ the induced automorphism on $H_{\nu} / H_{\nu^{\prime}}$. Suppose $h_{B}(\alpha, G)<\infty$ and $\lim _{\nu} h_{B}\left(\alpha^{\nu}, H_{\nu}\right)=\varepsilon>0$. (The limit exists since the net is nonincreasing.) Choose $\nu$ so that $h_{B}\left(\alpha^{\nu}, H_{\nu}\right)<3 / 2 \varepsilon$. By the first part of the proof, we can choose $\nu^{\prime}$ sufficiently large so that $h_{B}\left(\alpha_{\nu \nu}, H_{\nu} / H_{\nu}\right)$ is close to $h_{B}\left(\alpha_{\nu}, H_{\nu}\right)$, and in particular greater than $\varepsilon$. Since $h_{B}\left(\alpha^{\nu^{\prime}}, H_{\nu^{\prime}}\right)$ is at least $\varepsilon$, we have that

$$
\begin{aligned}
h_{B}\left(\alpha^{\nu}, H_{\nu}\right) & =h_{B}\left(\alpha^{\nu^{\prime}}, H_{\nu^{\prime}}\right)+h_{B}\left(\alpha_{\nu \nu^{\prime}}, H_{\nu} / H_{\nu^{\prime}}\right) \\
& >2 \varepsilon, \text { a contradiction . }
\end{aligned}
$$

Thus $h_{B}\left(\alpha^{\nu}, H_{\nu}\right) \rightarrow 0$, and $h_{B}\left(\alpha_{\nu}, G_{\nu}\right) \rightarrow h_{B}(\alpha, G)$.

If $h(\alpha, G)$ is infinite but $h_{B}\left(\alpha_{\nu}, G_{\nu}\right) \leqq M<\infty$ for all $\nu$, we similarly arrive at a contradiction. Once again, write

$$
h_{B}(\alpha, G)=h_{B}\left(\alpha_{\nu}, G_{\nu}\right)+h_{B}\left(\alpha^{\nu}, H_{\nu}\right) \text {, }
$$

which forces $h_{B}\left(\alpha^{\nu}, H_{\nu}\right)=\infty$ for all $\nu$. However for $\nu^{\prime} \geqq \nu$,

$$
h_{B}\left(\alpha_{\nu}, G_{\nu}\right)=h_{B}\left(\alpha_{\nu}, G_{\nu^{\prime}}\right)+h_{B}\left(\alpha_{\nu \nu^{\prime}}, H_{\nu} / H_{\nu^{\prime}}\right),
$$

and by the first part of the proof

$$
\lim _{\nu^{\prime}} h_{B}\left(\alpha_{\nu \nu^{\prime}}, H_{\nu} / H_{\nu^{\prime}}\right)=h_{B}\left(\alpha_{\nu}, H_{\nu}\right)=\infty .
$$

Hence $h_{B}\left(\alpha_{\nu}, G_{\nu}\right)$ is not bounded, and we have established

$$
h_{B}(\alpha, G)=\lim h_{B}\left(\alpha_{\nu}, G_{\nu}\right) \text {. }
$$

Finally, we must deal with $h_{\infty}$. But is easy, since

$$
\begin{aligned}
h_{\infty}(\alpha, G) & =h_{\infty}\left(\alpha_{\nu}, G_{\nu}\right)+h_{\infty}\left(\alpha^{\nu}, H_{\nu}\right) \\
& =h_{\infty}\left(\alpha_{\nu}, G_{\nu}\right),
\end{aligned}
$$

as $h_{\infty}$ vanishes on any compact group.

12.1. Some Questions. For any l.c.a. group $G, \operatorname{Aut}(G)$ is itself 
a topological group (though it need not be locally compact). The topology is described e.g., in [2] or [6]. Is the map $\alpha \in \operatorname{Aut}(G) \rightarrow$ $h(\alpha, G) \in[0, \infty]$ continuous? If $G$ is a compactly generated abelian Lie group, from the explicit formula for $h(\alpha, G)$ we can answer in the affirmative.

12.2. In view of Theorem 4 (vi) one might expect other relationships between the entropy of $\alpha \in \operatorname{Aut}(G)$ and $\hat{\alpha} \in \operatorname{Aut}(\Gamma)$ to hold. Even in the case of compact $G$ we do not know of any such results. If $G$ is compact and $\Gamma$ discrete, the adjoint $\hat{\alpha}$ of an $\alpha \epsilon$ $\operatorname{Aut}(G)$ extends to a homeomorphism $\bar{\alpha}$ of the Stone-Čech compactification $\bar{\Gamma}$. Is there a $\bar{\alpha}$-invariant measure $\omega$ on $\bar{\Gamma}$ for which the measure-theoretic entropy $h_{\omega}(\bar{\alpha}, \bar{\Gamma})$ equals the (Kolmogorov-Sinai) entropy $h(\alpha, G)$ ? We end the discussion with a counterexample which answers the question in the negative if we replace measuretheoretic entropy by topological entropy on $\bar{\Gamma}$ (see [7] for appropriate definitions).

Let $G=\prod_{i=-\infty}^{\infty}\left(Z_{n}\right)_{i}$, where $\left(Z_{p}\right)_{i}=Z_{p}$, the integers modulo $p$, for all $i \in Z$, and $\alpha \in \operatorname{Aut}(G)$ the bilateral shift. Then $h(\alpha, G)=\log p$.

Claim. The topological entropy of $\overline{\hat{\alpha}}$ on the Stone-Čech compactification $\bar{\Gamma}$ is infinite.

Proof. $\Gamma$ consists of all infinite sequences

$$
\bar{x}=\left(\cdots x_{k}, x_{k+1}, \cdots\right), x_{k} \in Z_{p} \quad \forall k \in Z
$$

and only finitely many of the $x_{k}$ 's are nonzero. We will say a sequence $\bar{x}$ begins at $k$ (resp. ends at $k$ ) if $x_{k} \neq 0$ and $x_{j}=0$ for $j<k$ (resp. $x_{k} \neq 0$ and $x_{j}=0$ for $j>k$ ); if $\bar{x}$ begins at $k_{1}$ and ends at $k_{2}$, we call $k_{2}-k_{1}+1$ the length of $\bar{x}$. Given an integer $N>0$ we will define a finite cover $\xi=\left\{E_{0}, E_{1}, \cdots, E_{N}\right\}$ of $\Gamma$. First we define inductively a sequence of integers $l_{j}$. Set $l_{0}=1$. Suppose, for some fixed $n$, that $l_{j}, j<n$, have been defined; set

$$
l_{n}=\inf \left\{l:(p-1)^{2} p^{l-2} \geqq N^{n} \text { and } l>l_{n-1}\right\} .
$$

There are $(p-1)^{2} p^{l-2}$ sequences of length $l(l \geqq 2)$ beginning at 0 . Set $m=N^{n}$. Thus it is possible to find $m$ distinct sequences $\bar{x}^{1, n}, \cdots, \bar{x}^{m, n}$ of length $l_{n}$, beginning at 0 . (How they are chosen is unimportant.) Let $F_{n}$ be any one-to-one function from the integers $\{1, \cdots, m\}$ onto the set of all sequences of the form $\left\{i_{1}, \cdots, i_{n}: i_{j} \in\{1, \cdots, N\}\right\}$. Let $\beta: \Gamma \rightarrow \Gamma$ be the bilateral shift; $\beta \bar{x}=\bar{y}$, where $y_{k}=x_{k+1}$. We now define subsets $E_{1}^{(n)}, \cdots, E_{N}^{(n)} \subset \Gamma$ as follows. We will put $\beta^{j} \bar{x}^{i, n}$ in $E_{i_{j+1}}^{(n)}, 0 \leqq j<n-1$, if and only if $F_{n}(i)=\left(i_{1}, \cdots, i_{n}\right), 1 \leqq i \leqq m$. 
Set $E_{i}=\bigcup_{n \geqq 1} E_{i}^{(n)}$ and $E_{0}=\Gamma \backslash \bigcup_{i=1}^{n} E_{i}$. Note that

$$
\begin{array}{r}
\bar{x}^{i, n} \in E_{i_{1}}^{(n)} \cap \beta^{-1} E_{i_{2}}^{(n)} \cap \cdots \cap \beta^{-(n-1)} E_{i_{n}}^{(n)} \\
\quad \subset E_{i_{1}} \cap \beta^{-1} E_{i_{2}} \cap \cdots \cap \beta^{-(n-1)} E_{i_{n}}
\end{array}
$$

if and only if $F_{n}(i)=\left(i_{1}, \cdots, i_{n}\right)$; furthermore, $\beta^{j} \bar{x}^{i, n} \notin E_{0}, 0 \leqq j<n$. Thus any covering of $\Gamma$ by $\xi \vee \beta^{-1} \xi \vee \cdots \vee \beta^{-(n-1)} \xi$ must contain at least $N^{n}$ sets.

Notice that $\beta$ is the transpose of the bilateral shift $\alpha$ on $G$. We embed $\Gamma$ in $\bar{\Gamma}$ in the usual way: if $A \subset \Gamma, \bar{A}$ is the set of all ultrafilters containing $A$. The operation $A \rightarrow \bar{A}$ respects finite unions and intersections, and it follows from the above that the topological entropy of $\overline{\hat{\alpha}}$ on $\bar{\Gamma}$ is at least $\log N$, for $N$ arbitrary, hence is infinite.

\section{REFERENCES}

1. R. Bowen, Entropy for group endomorphisms and homogeneous spaces, T.A.M.S., 523 (1971), 401-414.

2. E. Hewitt and K. Ross, Abstract Harmonic Analysis I, Springer-Verlag, New York, 1963.

3. M. W. Hirsch and C. C. Pugh, Stable manifolds and hyperbolic sets, Proceedings of Symposia in Pure Mathematics, Vol. XIV, 133-163, 1970.

4. S. Juzvinskii, Metric properties of endomorphisms of compact groups, A. M. S. Translations, Series 2, 66 (1968), 63-98.

5. J. Peters, Entropy on discrete abelian groups, Advances in Mathematics, 33 No.

1, (1979), 1-13.

6. J. Peters and T. Sund, Automorphisms of locally compact groups, Pacific J. Math., 76 No. 1, (1978), 143-156.

7. P. Walters, Ergodic Theory Introductory Lectures, Lectures Notes in Mathematics No. 458, Springer-Verlag, New York, 1975.

8. M. Weiss. Algebraic and other entropies of group endomorphisms, Mathematical Systems Theory, 8 No. 3, (1975), 243-248.

Received June 1, 1979 and in revised form June 6, 1980.

IOWA STATE UNIVERSity

AMES, IA 50011 


\section{PACIFIC JOURNAL OF MATHEMATICS}

\section{EDITORS}

DoNALD BABBITT (Managing Editor)

University of California

Los Angeles, CA 90024

Hugo Rossi

University of Utah

Salt Lake City, UT 84112

C. C. MOORE and ANDREw OGG

University of California

Berkeley, CA 94720
J. DugundJI

Department of Mathematics

University of Southern California

Los Angeles, CA 90007

R. FinN and J. Milgram

Stanford University

Stanford, CA 94305

ASSOCIATE EDITORS
R. ARENS
E. F. BECKENBACH
B. H. NeumanN
F. WOLF
K. YoshidA

\section{SUPPORTING INSTITUTIONS}

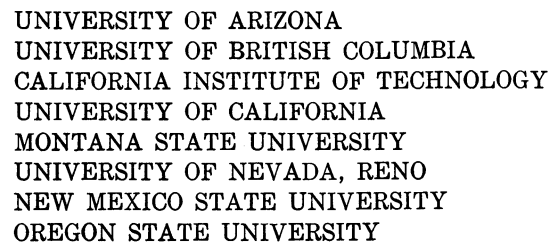

UNIVERSITY OF ARIZONA

UNIVERSITY OF BRITISH COLUMBIA

CALIFORNIA INSTITUTE OF TECHNOLOGY

UNIVERSITY OF CALIFORNIA

MONTANA STATE UNIVERSITY

UNIVERSITY OF NEVADA, RENO

NEW MEXICO STATE UNIVERSITY OREGON STATE UNIVERSITY

\author{
UNIVERSITY OF OREGON \\ UNIVERSITY OF SOUTHERN CALIFORNIA \\ STANFORD UNIVERSITY \\ UNIVERSITY OF HAWAII \\ UNIVERSITY OF TOKYO \\ UNIVERSITY OF UTAH \\ WASHINGTON STATE UNIVERSITY \\ UNIVERSITY OF WASHINGTON
}

The Supporting Institutions listed above contribute to the cost of publication of this Journal, but they are not owners or publishers and have no responsibility for its content or policies.

Mathematical papers intended for publication in the Pacific Journal of Mathematics should be in typed form or offset-reproduced, (not dittoed), double spaced with large margins. Please do not use built up fractions in the text of the manuscript. However, you may use them in the displayed equations. Underline Greek letters in red, German in green, and script in blue. The first paragraph or two must be capable of being used separately as a synopsis of the entire paper. Please propose a heading for the odd numbered pages of less than 35 characters. Manuscripts, in triplicate, may be sent to any one of the editors. Please classify according to the scheme of Math. Reviews, Index to Vol. 39. Supply name and address of author to whom proofs should be sent. All other communications should be addressed to the managing editor, or Elaine Barth, University of California, Los Angeles, California, 90024.

50 reprints to each author are provided free for each article, only if page charges have been substantially paid. Additional copies may be obtained at cost in multiples of 50 .

The Pacific Journal of Mathematics is issued monthly as of January 1966. Regular subscription rate: $\$ 102.00$ a year (6 Vols., 12 issues). Special rate: $\$ 51.00$ a year to individual members of supporting institutions.

Subscriptions, orders for numbers issued in the last three calendar years, and changes of address shoud be sent to Pacific Journal of Mathematics, P.O. Box 969, Carmel Valley, CA 93924, U.S.A. Old back numbers obtainable from Kraus Periodicals Co., Route 100, Millwood, NY 10546.

\footnotetext{
PUBLISHED BY PACIFIC JOURNAL OF MATHEMATICS, A NON-PROFIT CORPORATION

Printed at Kokusai Bunken Insatsusha (International Academic Printing Co., Ltd.). 8-8, 3-chome, Takadanobaba, Shinjuku-ku, Tokyo 160, Japan.
} 


\section{Pacific Journal of Mathematics}

\section{Vol. 96, No. 2 December, 1981}

Gerald A. Beer, A natural topology for upper semicontinuous functions and

a Baire category dual for convergence in measure $\ldots \ldots \ldots \ldots \ldots \ldots 251$

Georgia Benkart and J. Marshall Osborn, An investigation of real

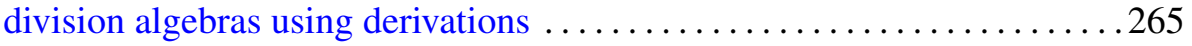

Donald Ian Cartwright and John R. McMullen, A structural criterion for the existence of infinite Sidon sets ........................ 301

Philip Hanlon, The fixed-point partition lattices $\ldots \ldots \ldots \ldots \ldots \ldots \ldots \ldots$

Eric Hayashi, The spectral density of a strongly mixing stationary Gaussian process

Chung-Wu Ho and Charles E. Morris, Jr., A graph-theoretic proof of

Sharkovsky's theorem on the periodic points of continuous functions . ...361

Sara Hurvitz, The automorphism groups of spaces and fibrations ....... 371

Atsushi Inoue, Schoichi Ota and Jun Tomiyama, Derivations of operator algebras into spaces of unbounded operators . .................. 389

Wolfgang B. Jurkat and Gary Sampson, On weak restricted estimates and endpoint problems for convolutions with oscillating kernels. I ........ 405

Georgios Koumoullis, Some topological properties of spaces of

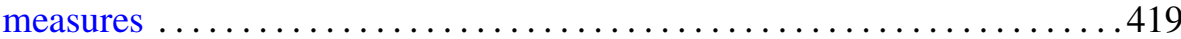

Wen Hsiung Lin, Algebraic Kahn-Priddy theorem ................. 435

Michael John McAsey, Invariant subspaces of nonselfadjoint crossed

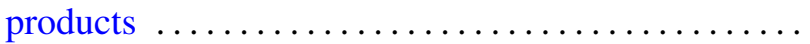

Justin Peters, Entropy of automorphisms on L.C.A. groups

Saburou Saitoh, A characterization of the adjoint $L$-kernel of Szegó type 